\title{
Chain Length of Amphipathic-Type Thioesters Dramatically Affects Reactivity in Aqueous Amidation Reactions with Cysteine Esters
}

\author{
Ikumi Otomo \\ Kanna Watanabe ${ }^{a}$ \\ Chiaki Kuroda*a \\ Kenichi Kobayashi*b \\ ${ }^{a}$ Department of Chemistry, Rikkyo University, Nishi-Ikebukuro, \\ Toshima-ku, Tokyo 171-8501, Japan \\ chkkuroda@rikkyo.ac.jp \\ b Graduate School of Pharmaceutical Sciences, Meiji Pharma- \\ ceutical University, Noshio, Kiyose, Tokyo 204-8588, Japan \\ kenichik@my-pharm.ac.jp
}
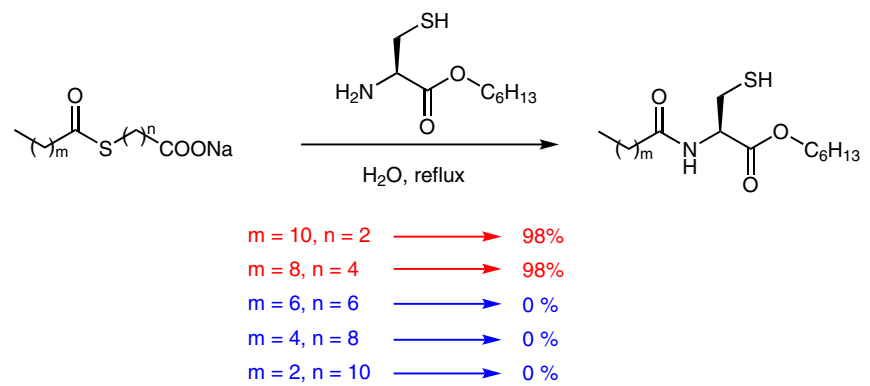

acid hexyl esters, but the reactions required reflux conditions due to the reduced nucleophilicity of the amine moiety. ${ }^{6}$

We recently compared the reactivities of amphipathic thioesters 1-5 $\left[\mathrm{CH}_{3}\left(\mathrm{CH}_{2}\right)_{m} \mathrm{COS}\left(\mathrm{CH}_{2}\right)_{n} \mathrm{COONa}, m+n=12\right]$ and reported that the position of the reaction site affects the amidation reaction. ${ }^{7}$ Compounds $\mathbf{1}$ and $\mathbf{2}$ reacted with various alkyl amines to afford amides in good yields, whereas amides were obtained with relatively low yields from $\mathbf{5}$ (Scheme 1). Amphipathic thioesters 1-5 form micelles in aqueous media, and the results suggested that the reaction occurs mainly on the micelle surface.

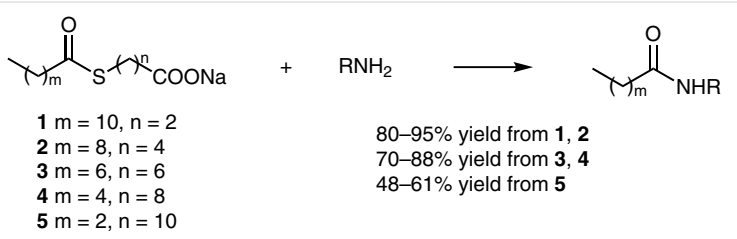

Scheme 1 Reaction of amphipathic thioesters with amines ${ }^{7}$

In the present study, to clarify the effect of side chain in the amino acid ester, we examined the reaction of 1-5 with both cysteine and valine esters. The formation of amides from cysteine derivatives is easier than from other amino acid derivatives because the reaction proceeds through a native chemical ligation (NCL) process. ${ }^{8}$ Herein, we report a dramatic difference in reactivity that is dependent on chain length in the reaction of amphipathic thioesters with cysteine esters.

Compounds 1-5 were prepared as previously reported ${ }^{7}$ and reacted with cysteine hexyl ester $(\mathbf{6})^{9}$ in water; the results are shown in Table $1 .^{10}$ All reactions were carried out at $20 \mathrm{mM} \mathrm{1-5}$, which is above the critical micelle concentration $(\mathrm{CMC})$ of each thioester. ${ }^{7}$ When $\mathbf{1}$ was reacted with $\mathbf{6}$ in water at room temperature for $24 \mathrm{~h}$, amide $\mathbf{7}^{11}$ was ob- 
tained in 96\% yield (entry 1). Compound 2 afforded $\mathbf{8}^{12}$ in $90 \%$ yield (entry 2 ). In contrast, 3-5 did not react with $\mathbf{6}$ (entries 3-5). The reactions of valine, leucine, isoleucine, and phenylalanine hexyl esters with $\mathbf{1}$ proceeded in refluxing water; 6 whereas the reaction of $\mathbf{6}$ with $\mathbf{1}$ and $\mathbf{2}$ proceeded at lower temperature.

A dramatic difference in yield was also observed when the reactions were carried out in refluxing water: $\mathbf{1}$ and $\mathbf{2}$ afforded amides $\mathbf{7}$ and $\mathbf{8}$, respectively, in almost quantitative yield (Table 1, entries 6 and 7); whereas 3-5 did not afford the corresponding amide (entries 8-10). The reactions were attempted using a higher concentration of $\mathbf{4}$ and $\mathbf{5}$, but no product was obtained (entries 11 and 12).

Table 1 Amidation Reaction of Thioesters 1-5 with Cysteine Hexyl Ester (6)

\begin{tabular}{|c|c|c|c|c|c|c|c|}
\hline & & 6 & & & & 7,8 & \\
\hline Entry & Thioester & $m$ & $n$ & Temp & Time (h) & Product & Yield (\%) \\
\hline 1 & 1 & 10 & 2 & r.t. & 24 & 7 & 96 \\
\hline 2 & 2 & 8 & 4 & r.t. & 24 & 8 & 90 \\
\hline 3 & 3 & 6 & 6 & r.t. & 24 & - & 0 \\
\hline 4 & 4 & 4 & 8 & r.t. & 24 & - & 0 \\
\hline 5 & 5 & 2 & 10 & r.t. & 24 & - & 0 \\
\hline 6 & 1 & 10 & 2 & reflux & 6 & 7 & 98 \\
\hline 7 & 2 & 8 & 4 & reflux & 6 & 8 & 98 \\
\hline 8 & 3 & 6 & 6 & reflux & 6 & - & 0 \\
\hline 9 & 4 & 4 & 8 & reflux & 6 & - & 0 \\
\hline 10 & 5 & 2 & 10 & reflux & 6 & - & 0 \\
\hline 11 & $4^{c}$ & 4 & 8 & reflux & 6 & - & 0 \\
\hline 12 & $5^{d}$ & 2 & 10 & reflux & 6 & - & 0 \\
\hline
\end{tabular}

${ }^{a}$ Concentration of 1-5 $=20 \mathrm{mM}$.

${ }^{\mathrm{b}}$ Isolated yield.

c Concentration of $\mathbf{4}=45 \mathrm{mM}$.

${ }^{d}$ Concentration of $\mathbf{5}=31 \mathrm{mM}$.

For reference, the reactions of 1-5 with valine hexyl ester $(\mathbf{9})^{6,9}$ were examined under the same conditions and the results are shown in Table 2 . No reaction occurred at room temperature (entries 1-5), whereas amides $\mathbf{1 0},{ }^{6} \mathbf{1 1},{ }^{13} \mathbf{1 2},{ }^{14}$ $\mathbf{1 3},{ }^{15}$ and $\mathbf{1 4},{ }^{16}$ were obtained in moderate yields from 1-5, respectively, after reflux (entries 6-10). The differences in yields between substrates were not pronounced.

Kawabata and Kinoshita reported differences in the reaction of ethyl thioesters and dodecyl thioesters with thioamino acids. ${ }^{5}$ Compounds $\mathbf{1 - 5}$ were treated with cysteine ethyl ester $(\mathbf{1 5})^{9}$ and their reaction with hexyl ester $\mathbf{6}$ was compared (Table 3). Only $\mathbf{1}$ afforded the corresponding am-
Table 2 Amidation Reaction of Thioesters 1-5 with Valine Hexyl Ester (9)

\begin{tabular}{lllllllll} 
& & & & & & & \\
\hline
\end{tabular}

ide 16, ${ }^{17}$ in $73 \%$ yield (entry 1 ). Compound 2 afforded a trace amount of amide (entry 2), and 3-5 did not afford amide (entries 3-5).

Table 3 Amidation Reaction of Thioesters 1-5 with Cysteine Ethyl Ester (15)

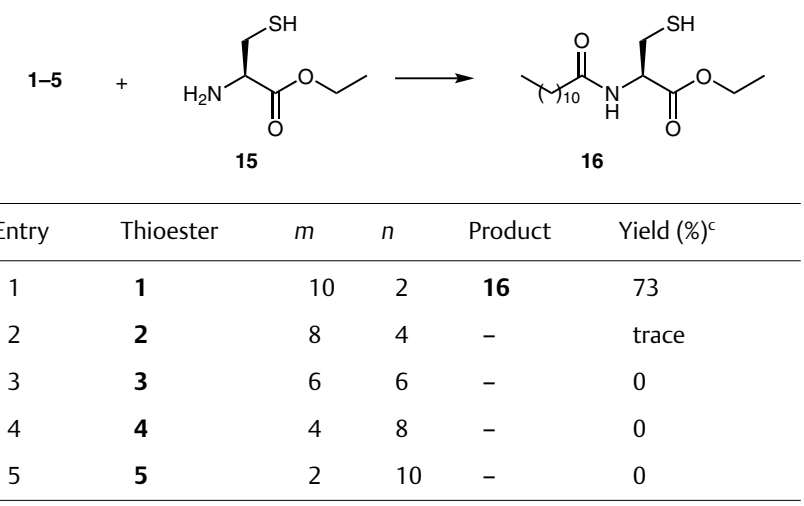

a Concentration of $\mathbf{1 - 5}=20 \mathrm{mM}$.

${ }^{\mathrm{b}}$ All reactions were carried out in refluxing water for $6 \mathrm{~h}$. c Isolated yield.

Compound 2 gave different results in the reaction with cysteine hexyl ester $(\mathbf{6})$ and ethyl ester (15). Thus, the reaction was further examined by using butyl ester $17^{9}$ (Table 4). Compound 1 afforded the corresponding ester $\mathbf{1 8}^{18}$ in 89 $\%$ yield, as expected (entry 1 ). Compound 2 afforded $19^{19}$ in low yield (entry 2 ), suggesting that the reaction was slow. Indeed, after longer reaction time, $\mathbf{1 9}$ was obtained in good yield (entry 3 ). 
Table 4 Amidation Reaction of Thioesters 1 and $\mathbf{2}$ with Cysteine Butyl Ester (17) ${ }^{\mathrm{a}, \mathrm{b}}$

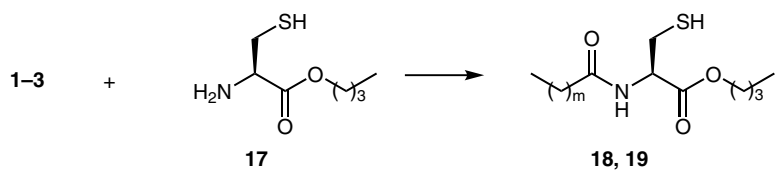

\begin{tabular}{lllllll}
\hline Entry & Thioester & $m$ & $n$ & Time (h) & Product & Yield $(\%)^{c}$ \\
\hline 1 & $\mathbf{1}$ & 10 & 2 & 6 & $\mathbf{1 8}$ & 89 \\
2 & $\mathbf{2}$ & 8 & 4 & 6 & $\mathbf{1 9}$ & 49 \\
3 & $\mathbf{2}$ & 8 & 4 & 12 & $\mathbf{1 9}$ & 96 \\
\hline
\end{tabular}

a Concentration of $\mathbf{1 - 5}=20 \mathrm{mM}$.

${ }^{\mathrm{b}}$ All reactions were carried out in refluxing water.

' Isolated yield.

The difference in yield based on the position of the reaction site in long-chain amphipathic-type thioesters 1-5 was distinct for cysteine esters, whereas no such difference was observed for valine ester. The difference was moderate for $n$-alkyl amines. ${ }^{7}$ These results indicate that the difference in yield is affected by not only the position of the reaction site (thioester group), but also by the nature of the amino acid side chain. Cysteine esters form a zwitterionic structure under the reaction conditions ( $\mathrm{pH}$ ca. 8.5). Due to this highly hydrophilic moiety, the reaction site of $\mathbf{6}$ (the thiolate moiety) is restricted to the micelle surface and thus the two reaction sites (the thiolate and thioester moieties) are in close proximity in the reaction of $\mathbf{1}$ and $\mathbf{2}$ (Figure 1a) but distant in 3-5 (Figure 1b). In contrast, valine hexyl ester $\mathbf{9}$ can penetrate the micelle due to the presence of a hydrophobic isopropyl group, resulting in the formation of amides from thioesters 1-5 (Table 2).

Differences were also observed in the reaction of $\mathbf{2}$ with cysteine esters. The yield of amide was $98 \%$ (at reflux temperature) using hexyl ester $\mathbf{6}$, whereas only a trace amount of product was detected in the reaction with ethyl ester $\mathbf{1 5 .}$ Compound $\mathbf{1 5}$ is water soluble, and its ethyl group is too short to provide a hydrophobic effect. Consequently, 15 probably remains mainly in the bulk water and thus reacts only with $\mathbf{1}$, the reaction site of which locates on the micelle surface. Slow reaction of $\mathbf{2}$ and $\mathbf{1 7}$ suggests that hydrophobic interaction with butyl group is limited.

In conclusion, a dramatic difference in yield based on the chain length of amphipathic-type thioesters was observed when reacted with cysteine esters in water. In the reaction of a micelle-forming thioester and an amino acid ester, both the position of the reaction site in the thioester and the hydrophilic/hydrophobic nature of the amino acid side chain are important factors. The present findings may be applied to substrate-specific reactions.
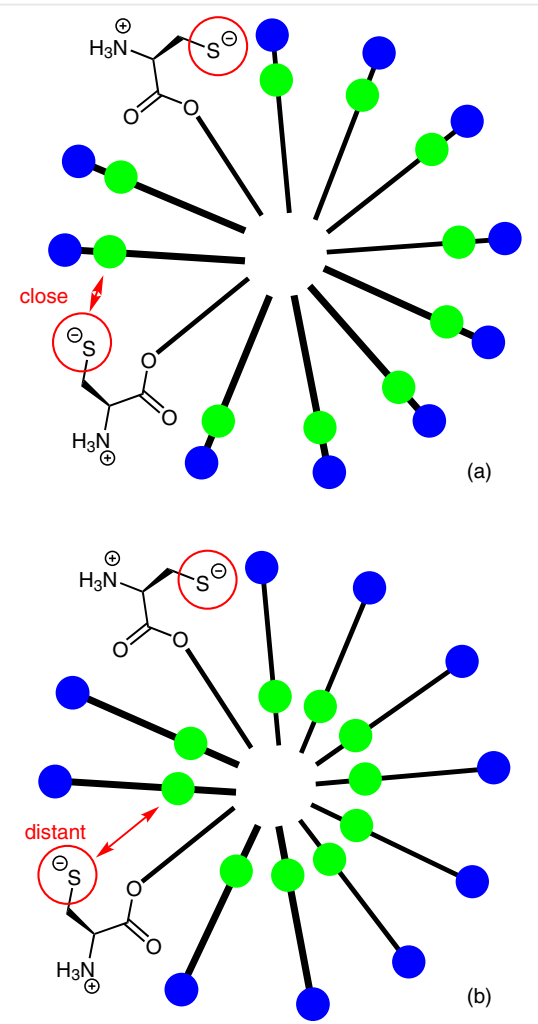

Figure 1 Micellar model of the reaction. Blue circle = hydrophilic group $\left(\mathrm{COO}^{-} \mathrm{Na}^{+}\right)$, green circle $=$reaction site (thioester).

\section{Funding Information}

This work was partly supported by the Strategic Research Foundation Grant-aided Projects for Private Universities from the Ministry of Education, Culture, Sports, Science, and Technology, Japan.

\section{References and Notes}

(1) (a) Organic Reactions in Water; Lindström, U. M., Ed.; Blackwell Publishing: Oxford, 2007. (b) Li, C.-J. Chem. Rev. 1993, 93, 2023. (c) Li, C.-J.; Chen, L. Chem. Soc. Rev. 2006, 35, 68. (d) Lindström, U. M. Chem. Rev. 2002, 102, 2751. (e) Kobayashi, S. Pure Appl. Chem. 2013, 85, 1089. (f) Chanda, A.; Fokin, V. V. Chem. Rev. 2009, 109, 725.

(2) (a) Breslow, R. Acc. Chem. Res. 1991, 24, 159. (b) Breslow, R.; Groves, K.; Mayer, M. U. Pure Appl. Chem. 1998, 70, 1933. (c) Otto, S.; Engberts, J. B. F. N. Org. Biomol. Chem. 2003, 1, 2809.

(3) (a) Engberts, J. B. F. N. Pure Appl. Chem. 1995, 67, 823. (b) Otto, S.; Engberts, J. B. F. N.; Kwak, J. C. T. J. Am. Chem. Soc. 1998, 120, 9517.

(4) Dwars, T.; Paetzold, E.; Oehme, G. Angew. Chem. Int. Ed. 2005, $44,7174$.

(5) (a) Kawabata, Y.; Kinoshita, M. Makromol. Chem. 1974, 175, 105. (b) Kawabata, Y.; Kinoshita, M. Makromol. Chem. 1975, 176, 49. (c) Kawabata, Y.; Kinoshita, M. Makromol. Chem. 1975, 176, 2797. (d) Kawabata, Y.; Kinoshita, M. Makromol. Chem. 1975, 176, 2807.

(6) Torihata, A.; Kuroda, C. Synlett 2011, 2035. 
(7) Otomo, I.; Kuroda, C. Adv. Chem. Eng. Sci. 2015, 5, 311.

(8) Dawson, P. E.; Muir, T. W.; Clark-Lewis, I.; Kent, S. B. H. Science 1994, 266, 776.

(9) Compounds $\mathbf{6}$ and $\mathbf{9}$ were prepared by TfOH-catalyzed esterification of cysteine or valine, respectively, with 1-hexanol. Compound 17 was prepared in the same way with 1-butanol. Compound $\mathbf{1 5}$ is commercially available as the hydrochloride, and $\mathrm{HCl}$ was removed by the addition of $\mathrm{NaOH} / \mathrm{MeOH}$, followed by recrystallization $\left(\mathrm{H}_{2} \mathrm{O} / \mathrm{MeCN}\right)$.

(10) In a typical experiment, thioester $1(44.3 \mathrm{mg}, 0.142 \mathrm{mmol})$ was added to a stirred mixture of $\mathbf{6}(59.5 \mathrm{mg}, 0.290 \mathrm{mmol})$ in water $(6 \mathrm{~mL})$, and the mixture was heated to reflux for $6 \mathrm{~h}$. The mixture was diluted with water, extracted with EtOAc, and the organic layer washed with aqueous $\mathrm{NaHCO}_{3}$ solution and dried over $\mathrm{Na}_{2} \mathrm{SO}_{4}$. Filtration and evaporation of the solvent afforded 7 (53.8 $\mathrm{mg}, 0.139 \mathrm{mmol}, 98 \%)$. In the same manner, 2 (50.4 mg, $0.162 \mathrm{mg}$ ) was reacted with 6 ( $72.5 \mathrm{mg}, 0.354 \mathrm{mmol})$ to afford 8 (56.9 mg, $0.158 \mathrm{mmol}, 98 \%$ ). Reaction of $\mathbf{2}(20.8 \mathrm{mg}$ ) and $\mathbf{9}$ (32.2 $\mathrm{mg}$ ) afforded 11 (13.8 mg, $58 \%$ ) after silica gel column chromatography (hexane/EtOAc); 3 (15.7 $\mathrm{mg}$ ) and $\mathbf{9}(27.1 \mathrm{mg}$ ) afforded 12 (12.0 mg, 73\%); 4 (13.3 mg) and $\mathbf{9}(21.9 \mathrm{mg})$ afforded 13 (7.0 $\mathrm{mg}, 55 \%) ; \mathbf{5}(97.7 \mathrm{mg})$ and $\mathbf{9}(131.2 \mathrm{mg})$ afforded $\mathbf{1 4}(38.9 \mathrm{mg}$, 46\%); 1 (63.2 mg) and 17 (73.4 mg) afforded 18 (64.9 mg, 89\%); $\mathbf{2}(28.1 \mathrm{mg})$ and $\mathbf{1 7}(33.5 \mathrm{mg})$ afforded $\mathbf{1 9}(29.0 \mathrm{mg}, 96 \%$, refluxing for $12 \mathrm{~h}$ )

(11) Compound 7: $\mathrm{Mp} 65.6-67.5^{\circ} \mathrm{C}$; IR (KBr): $3324,1736,1670 \mathrm{~cm}^{-1}$; ${ }^{1} \mathrm{H} \operatorname{NMR}\left(\mathrm{CDCl}_{3}\right): \delta=0.88\left(\mathrm{t}, J=6.5 \mathrm{~Hz}, 3 \mathrm{H}, \mathrm{CH}_{3}\right), 0.90(\mathrm{t}, J=6.2$ $\left.\mathrm{Hz}, 3 \mathrm{H}, \mathrm{CH}_{3}\right), 1.22-1.39\left(\mathrm{~m}, 22 \mathrm{H}, 11 \times \mathrm{CH}_{2}\right), 1.57-1.73(\mathrm{~m}, 4 \mathrm{H}$, $\left.2 \times \mathrm{CH}_{2}\right), 2.25\left(\mathrm{t}, J=7.5 \mathrm{~Hz}, 2 \mathrm{H}, \mathrm{NHCOCH}_{2}\right), 3.17(\mathrm{dd}, J=5.2,14.0$ $\mathrm{Hz}, 1 \mathrm{H}, \mathrm{CHHSH}), 3.23$ (dd, J = 4.9, $14.0 \mathrm{~Hz}, 1 \mathrm{H}, \mathrm{CHHSH}), 4.08-$ $4.24\left(\mathrm{~m}, 2 \mathrm{H}, \mathrm{COOCH}_{2}\right), 4.86(\mathrm{td}, J=5.0,7.1 \mathrm{~Hz}, 1 \mathrm{H}, \mathrm{CONHCH})$, $6.47(\mathrm{~d}, J=7.2 \mathrm{~Hz}, 1 \mathrm{H}, \mathrm{NH}) ;{ }^{13} \mathrm{C}$ NMR $\left(\mathrm{CDCl}_{3}\right): \delta=14.0,14.1$, $22.5,22.7,25.5,28.4,29.3,29.3,29.4,29.5,29.6,29.6(2 \mathrm{C}), 31.3$, $31.9,36.5,41.0,51.7,66.2,170.6,173.1$; MS (FAB): $m / z=388$ $[\mathrm{M}+\mathrm{H}]^{+}, 119$; HRMS-FAB: $\mathrm{m} / z[\mathrm{M}+\mathrm{H}]^{+}$calcd for $\mathrm{C}_{21} \mathrm{H}_{42} \mathrm{NO}_{3} \mathrm{~S}$ : 388.2885; found: 388.2883

(12) Compound 8: $\mathrm{Mp} 71.4-73.0{ }^{\circ} \mathrm{C}$; IR (KBr): 3313, 1731, $1644 \mathrm{~cm}^{-}$ ${ }^{1}$; ${ }^{1} \mathrm{H} \mathrm{NMR}\left(\mathrm{CDCl}_{3}\right): \delta=0.88\left(\mathrm{t}, J=6.8 \mathrm{~Hz}, 3 \mathrm{H}, \mathrm{CH}_{3}\right), 0.90(\mathrm{t}, J=6.8$ $\left.\mathrm{Hz}, 3 \mathrm{H}, \mathrm{CH}_{3}\right), 1.23-1.38\left(\mathrm{~m}, 18 \mathrm{H}, 9 \times \mathrm{CH}_{2}\right), 1.59-1.70(\mathrm{~m}, 4 \mathrm{H}$, $2 \times \mathrm{CH}_{2}$ ), 2.26 (app. t, $J=7.5 \mathrm{~Hz}, 2 \mathrm{H}, \mathrm{NHCOCH}_{2}$ ), 3.17 (dd, $J=5.3$, $14.0 \mathrm{~Hz}, 1 \mathrm{H}, \mathrm{CHHSH}), 3.24$ (dd, $J=5.0,14.0 \mathrm{~Hz}, 1 \mathrm{H}, \mathrm{CHHSH}$ ), 4.09-4.21 (m, $\left.2 \mathrm{H}, \mathrm{COOCH}_{2}\right), 4.86(\mathrm{td}, J=5.0,7.3 \mathrm{~Hz}, 1 \mathrm{H}, \mathrm{CON}-$ $\mathrm{HCH}), 6.51$ (d, $J=7.2 \mathrm{~Hz}, 1 \mathrm{H}, \mathrm{NH}) ;{ }^{13} \mathrm{C} \mathrm{NMR}\left(\mathrm{CDCl}_{3}\right): \delta=14.0$, 14.1, 22.5, 22.7, 25.5 (2C), 28.4, 29.3 (2C), 29.4, 29.5, 31.3, 31.9, $36.5,40.9,51.7,66.2,170.6,173.1$; MS (FAB): $m / z=360[\mathrm{M}+\mathrm{H}]^{+}$, 119; HRMS-FAB: $m / z[\mathrm{M}+\mathrm{H}]^{+}$calcd for $\mathrm{C}_{19} \mathrm{H}_{38} \mathrm{NO}_{3} \mathrm{~S}: 360.2572$; found: 360.2569

(13) Compound 11: Oil; IR (neat): 3308, 1742, 1651, $1539 \mathrm{~cm}^{-1} ;{ }^{1} \mathrm{H}$ $\operatorname{NMR}\left(\mathrm{CDCl}_{3}\right): \delta=0.88\left(\mathrm{t}, J=6.8 \mathrm{~Hz}, 3 \mathrm{H}, \mathrm{CH}_{3}\right), 0.89(\mathrm{t}, J=6.8 \mathrm{~Hz}$, $\left.3 \mathrm{H}, \mathrm{CH}_{3}\right), 0.90\left(\mathrm{~d}, J=6.9 \mathrm{~Hz}, 3 \mathrm{H}, \mathrm{CH}_{3}\right), 0.94(\mathrm{~d}, J=6.9 \mathrm{~Hz}, 3 \mathrm{H}$, $\left.\mathrm{CH}_{3}\right), 1.23-1.40\left(\mathrm{~m}, 18 \mathrm{H}, 9 \times \mathrm{CH}_{2}\right), 1.58-1.69\left(\mathrm{~m}, 4 \mathrm{H}, 2 \times \mathrm{CH}_{2}\right)$, 2.10-2.22 (m, $\left.1 \mathrm{H}, \mathrm{C} \underline{\mathrm{H}}\left(\mathrm{CH}_{3}\right)_{2}\right), 2.23$ (app. t, $J=7.6 \mathrm{~Hz}, 2 \mathrm{H}, \mathrm{NHCO}-$ $\left.\mathrm{CH}_{2}\right), 4.07-4.18\left(\mathrm{~m}, 2 \mathrm{H}, \mathrm{COOCH}_{2}\right), 4.59(\mathrm{dd}, J=8.7,4.8 \mathrm{~Hz}, 1 \mathrm{H}$, CHiPr), 6.00 (br d, $J=8.7 \mathrm{~Hz}, 1 \mathrm{H}, \mathrm{NH}) ;{ }^{13} \mathrm{C} \mathrm{NMR}\left(\mathrm{CDCl}_{3}\right): \delta=13.9$, 14.1, 17.7, 18.9, 22.5, 22.6, 25.5, 25.7, 28.5, 29.2 (2C), 29.3, 29.4, 31.3, 31.4, 31.8, 36.8, 56.7, 65.4, 172.4, 173.0; MS (EI, $70 \mathrm{eV}$ ): $m / z=355\left[\mathrm{M}^{+}\right], 226$; HRMS: $m / z\left[\mathrm{M}^{+}\right]$calcd for $\mathrm{C}_{21} \mathrm{H}_{41} \mathrm{NO}_{3}$ : 355.3086; found: 355.3090
(14) Compound 12: Oil; IR (neat): 3308, 1740, 1734, 1653, $1541 \mathrm{~cm}^{-1}$; ${ }^{1} \mathrm{H}$ NMR $\left(\mathrm{CDCl}_{3}\right): \delta=0.88\left(\mathrm{t}, J=6.9 \mathrm{~Hz}, 3 \mathrm{H}, \mathrm{CH}_{3}\right), 0.90(\mathrm{t}, J=6.8$ $\left.\mathrm{Hz}, 3 \mathrm{H}, \mathrm{CH}_{3}\right), 0.90\left(\mathrm{~d}, J=6.8 \mathrm{~Hz}, 3 \mathrm{H}, \mathrm{CH}_{3}\right), 0.94(\mathrm{~d}, J=6.9 \mathrm{~Hz}, 3 \mathrm{H}$, $\left.\mathrm{CH}_{3}\right), 1.23-1.40\left(\mathrm{~m}, 14 \mathrm{H}, 7 \times \mathrm{CH}_{2}\right), 1.55-1.70\left(\mathrm{~m}, 4 \mathrm{H}, 2 \times \mathrm{CH}_{2}\right)$, 2.10-2.21 (m, $\left.1 \mathrm{H}, \mathrm{CH}\left(\mathrm{CH}_{3}\right)_{2}\right), 2.24$ (app. t, J = 7.6 Hz, $2 \mathrm{H}, \mathrm{NHCOCH}_{2}$ ), 4.07-4.18 (m, $\left.2 \mathrm{H}, \mathrm{COOCH}_{2}\right), 4.58(\mathrm{dd}, J=8.7,4.7 \mathrm{~Hz}, 1 \mathrm{H}, \mathrm{C} \underline{\mathrm{H} i P r})$, $5.92(\mathrm{br} \mathrm{d}, J=8.8 \mathrm{~Hz}, 1 \mathrm{H}, \mathrm{NH}) ;{ }^{13} \mathrm{C} \mathrm{NMR}\left(\mathrm{CDCl}_{3}\right): \delta=14.0,14.0$, 17.7, 18.9, 22.5, 22.6, 25.5, 25.7, 28.5, 29.0, 29.2, 31.3, 31.4, 31.7, 36.8, 56.7, 65.4, 172.3, 173.0; MS (EI, $70 \mathrm{eV}$ ): $m / z=327\left[\mathrm{M}^{+}\right]$, 198; HRMS: $m / z\left[\mathrm{M}^{+}\right]$calcd for $\mathrm{C}_{19} \mathrm{H}_{27} \mathrm{NO}_{3}$ : 327.2773; found: 327.2769.

(15) Compound 13: Oil; IR (neat): 3304, 1742, 1651, $1539 \mathrm{~cm}^{-1} ;{ }^{1} \mathrm{H}$ $\operatorname{NMR}\left(\mathrm{CDCl}_{3}\right): \delta=0.90\left(\mathrm{t}, J=6.8 \mathrm{~Hz}, 6 \mathrm{H}, 2 \times \mathrm{CH}_{3}\right), 0.90(\mathrm{~d}, J=6.9$ $\left.\mathrm{Hz}, 3 \mathrm{H}, \mathrm{CH}_{3}\right), 0.94\left(\mathrm{~d}, \mathrm{~J}=6.9 \mathrm{~Hz}, 3 \mathrm{H}, \mathrm{CH}_{3}\right), 1.24-1.40(\mathrm{~m}, 10 \mathrm{H}$, $\left.5 \times \mathrm{CH}_{2}\right), 1.56-1.71\left(\mathrm{~m}, 4 \mathrm{H}, 2 \times \mathrm{CH}_{2}\right), 2.11-2.21(\mathrm{~m}, 1 \mathrm{H}$, $\left.\mathrm{CH}\left(\mathrm{CH}_{3}\right)_{2}\right), 2.24$ (app. t, $\left.J=7.7 \mathrm{~Hz}, 2 \mathrm{H}, \mathrm{NHCOCH}_{2}\right), 4.07-4.19(\mathrm{~m}$, $2 \mathrm{H}, \mathrm{COOCH}_{2}$ ), 4.58 (dd, $J=8.9,4.8 \mathrm{~Hz}, 1 \mathrm{H}, \mathrm{CHiPr}$ ), 5.93 (br d, $J=$ $8.8 \mathrm{~Hz}, 1 \mathrm{H}, \mathrm{NH}) ;{ }^{13} \mathrm{C}$ NMR $\left(\mathrm{CDCl}_{3}\right): \delta=13.9,14.0,17.8,18.9$, $22.4,22.5,25.4,25.5,28.5,31.3,31.4(2 \mathrm{C}), 36.7,56.8,65.4$, 172.4, 173.0; MS (EI, $70 \mathrm{eV}): m / z=299$ [M+1, 170; HRMS: $m / z$ $\left[\mathrm{M}^{+}\right]$calcd for $\mathrm{C}_{17} \mathrm{H}_{33} \mathrm{NO}_{3}: 299.2460$; found: 299.2458

(16) Compound 14: Oil; IR (neat): 3308, 1744, 1734, 1653, $1541 \mathrm{~cm}^{-1}$; ${ }^{1} \mathrm{H} \mathrm{NMR}\left(\mathrm{CDCl}_{3}\right): \delta=0.90\left(\mathrm{t}, J=7.0 \mathrm{~Hz}, 3 \mathrm{H}, \mathrm{CH}_{3}\right), 0.90(\mathrm{~d}, J=6.9$ $\left.\mathrm{Hz}, 3 \mathrm{H}, \mathrm{CH}_{3}\right), 0.95\left(\mathrm{~d}, J=6.9 \mathrm{~Hz}, 3 \mathrm{H}, \mathrm{CH}_{3}\right), 0.96(\mathrm{t}, J=7.3 \mathrm{~Hz}, 3 \mathrm{H}$, $\left.\mathrm{CH}_{3}\right), 1.24-1.40\left(\mathrm{~m}, 6 \mathrm{H}, 3 \times \mathrm{CH}_{2}\right), 1.54-1.74\left(\mathrm{~m}, 4 \mathrm{H}, 2 \times \mathrm{CH}_{2}\right)$, 2.10-2.22 (m, $\left.1 \mathrm{H}, \mathrm{CH}\left(\mathrm{CH}_{3}\right)_{2}\right), 2.23$ (app. t, J = 7.5 Hz, $2 \mathrm{H}, \mathrm{NHCOCH}_{2}$ ), 4.07-4.19 (m, $\left.2 \mathrm{H}, \mathrm{COOCH}_{2}\right), 4.59$ (dd, $\left.J=8.8,4.7 \mathrm{~Hz}, 1 \mathrm{H}, \mathrm{CHiPr}\right)$, $5.92(\mathrm{br} \mathrm{d}, J=8.2 \mathrm{~Hz}, 1 \mathrm{H}, \mathrm{NH}) ;{ }^{13} \mathrm{C} \mathrm{NMR}\left(\mathrm{CDCl}_{3}\right): \delta=13.7,14.0$, 17.7, 18.9, 19.1, 22.5, 25.5, 28.5, 31.3, 31.4, 38.7, 56.7, 65.4, 172.4, 172.8; MS (EI, $70 \mathrm{eV}): m / z=271\left[\mathrm{M}^{+}\right], 142$; HRMS: $m / z$ $\left[\mathrm{M}^{+}\right]$calcd for $\mathrm{C}_{15} \mathrm{H}_{29} \mathrm{NO}_{3}: 271.2147$; found: 271.2144

(17) Kimura, K.; Mori, M.; Matsuo, T. Jpn Kokai Tokkyo Koho 53104741, 1978; Chem. Abstr. 1979, 90, 28889.

(18) Compound 18: $\mathrm{Mp} 63.0-64.5{ }^{\circ} \mathrm{C}$; IR (KBr): 3310, 1728, 1641, $1545 \mathrm{~cm}^{-1} ;{ }^{1} \mathrm{H}$ NMR $\left(\mathrm{CDCl}_{3}\right): \delta=0.88\left(\mathrm{t}, J=6.7 \mathrm{~Hz}, 3 \mathrm{H}, \mathrm{CH}_{3}\right)$, $0.94\left(\mathrm{t}, J=7.3 \mathrm{~Hz}, 3 \mathrm{H}, \mathrm{CH}_{3}\right), 1.23-1.44\left(\mathrm{~m}, 18 \mathrm{H}, 9 \times \mathrm{CH}_{2}\right), 1.59-$ $1.71\left(\mathrm{~m}, 4 \mathrm{H}, 2 \times \mathrm{CH}_{2}\right), 2.26\left(\mathrm{t}, J=7.6 \mathrm{~Hz}, 2 \mathrm{H}, \mathrm{NHCOCH}_{2}\right), 3.17$ (dd, $J=5.2,14.1 \mathrm{~Hz}, 1 \mathrm{H}, \mathrm{CHHSH}), 3.24(\mathrm{dd}, J=4.8,14.1 \mathrm{~Hz}, 1 \mathrm{H}$, CHHSH $), 4.13-4.25\left(\mathrm{~m}, 2 \mathrm{H}, \mathrm{COOCH}_{2}\right), 4.86(\mathrm{td}, J=5.0,7.2 \mathrm{~Hz}, 1$ $\mathrm{H}, \mathrm{CONHCH}), 6.47(\mathrm{~d}, J=7.3 \mathrm{~Hz}, 1 \mathrm{H}, \mathrm{NH}) ;{ }^{13} \mathrm{C} \mathrm{NMR}\left(\mathrm{CDCl}_{3}\right): \delta=$ 13.7, 14.1, 19.1, 22.7, 25.5, 29.3 (2C), 29.4, 29.5, 29.6 (2C), 30.4, 31.9, 36.5, 40.9, 51.7, 65.9, 170.6, 173.1; MS (EI, $70 \mathrm{eV}): \mathrm{m} / z=$ $359\left[\mathrm{M}^{+}\right], 200$; HRMS: $m / z\left[\mathrm{M}^{+}\right]$calcd for $\mathrm{C}_{19} \mathrm{H}_{37} \mathrm{NO}_{3} \mathrm{~S}$ : 359.2496; found: 359.2489

(19) Compound 19: $\mathrm{Mp} \mathrm{69.0-71.0}{ }^{\circ} \mathrm{C}$; IR (KBr): 3314, 1728, 1641, $1545 \mathrm{~cm}^{-1} ;{ }^{1} \mathrm{H}$ NMR $\left(\mathrm{CDCl}_{3}\right): \delta=0.88\left(\mathrm{t}, J=6.7 \mathrm{~Hz}, 3 \mathrm{H}, \mathrm{CH}_{3}\right)$, $0.94\left(\mathrm{t}, J=7.3 \mathrm{~Hz}, 3 \mathrm{H}, \mathrm{CH}_{3}\right), 1.23-1.44\left(\mathrm{~m}, 14 \mathrm{H}, 7 \times \mathrm{CH}_{2}\right), 1.58-$ $1.75\left(\mathrm{~m}, 4 \mathrm{H}, 2 \times \mathrm{CH}_{2}\right.$ ), 2.26 (app. t, $J=7.5 \mathrm{~Hz}, 2 \mathrm{H}, \mathrm{NHCOCH}_{2}$ ), $3.17(\mathrm{dd}, J=5.0,14.0 \mathrm{~Hz}, 1 \mathrm{H}, \mathrm{CHHSH}), 3.24(\mathrm{dd}, J=4.9,14.0 \mathrm{~Hz}$, $1 \mathrm{H}, \mathrm{CHHSH}), 4.11-4.25\left(\mathrm{~m}, 2 \mathrm{H}, \mathrm{COOCH}_{2}\right), 4.86$ (td, $J=5.0,7.3$ $\mathrm{Hz}, 1 \mathrm{H}, \mathrm{CONHCH}), 6.52$ (d, $J=7.1 \mathrm{~Hz}, 1 \mathrm{H}, \mathrm{NH}) ;{ }^{13} \mathrm{C} \mathrm{NMR}\left(\mathrm{CDCl}_{3}\right)$ : $\delta=13.6,14.1,19.1,22.6,25.5,29.3$ (3C), 29.4, 30.4, 31.8, 36.4, 40.9, 51.7, 65.8, 170.6, 173.1; MS (EI, $70 \mathrm{eV}) \mathrm{m} / z=331\left[\mathrm{M}^{+}\right]$, 172; HRMS: $m / z$ [M+] calcd for $\mathrm{C}_{17} \mathrm{H}_{33} \mathrm{NO}_{3} \mathrm{~S}$ : 331.2183 ; found: 331.2175. 